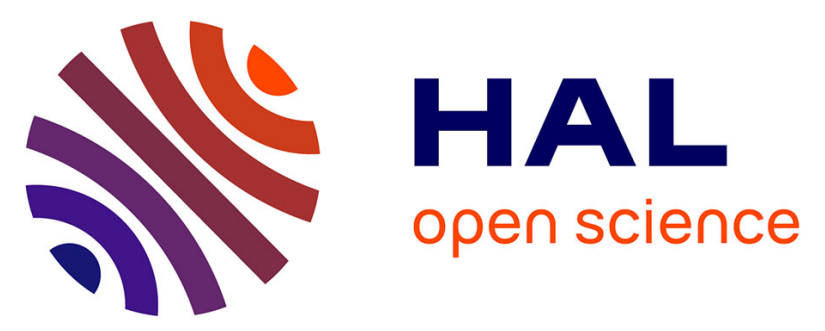

\title{
Large-volume excitation of air, argon, nitrogen and combustible mixtures by thermal jets produced by nanosecond spark discharges
}

Sergey Stepanyan, Jun Hayashi, Arthur Salmon, Gabi D. Stancu, Christophe

O Laux

\section{To cite this version:}

Sergey Stepanyan, Jun Hayashi, Arthur Salmon, Gabi D. Stancu, Christophe O Laux. Large-volume excitation of air, argon, nitrogen and combustible mixtures by thermal jets produced by nanosecond spark discharges. Plasma Sources Science and Technology, 2017, 26 (4), 10.1088/1361-6595/aa5a2b . hal-01866231

\section{HAL Id: hal-01866231 \\ https://hal.science/hal-01866231}

Submitted on 3 Sep 2018

HAL is a multi-disciplinary open access archive for the deposit and dissemination of scientific research documents, whether they are published or not. The documents may come from teaching and research institutions in France or abroad, or from public or private research centers.
L'archive ouverte pluridisciplinaire HAL, est destinée au dépôt et à la diffusion de documents scientifiques de niveau recherche, publiés ou non, émanant des établissements d'enseignement et de recherche français ou étrangers, des laboratoires publics ou privés. 


\title{
Large-volume excitation of air, argon, nitrogen and combustible mixtures by thermal jets produced by nanosecond spark discharges
}

\author{
Sergey Stepanyan ${ }^{1}$, Jun Hayashi ${ }^{2}$, Arthur Salmon ${ }^{1}$, Gabi D. Stancu ${ }^{1}$, Christophe O. Laux ${ }^{1}$ \\ ${ }^{1}$ Laboratoire EM2C, CNRS UPR288, Centrale Supélec, Université Paris Saclay, Grande Voie des Vignes, 92295 \\ Chatenay-Malabry, France \\ ${ }^{2}$ Osaka University, 1-1 Yamadaoka, Suita, 565-0871, Japan
}

\begin{abstract}
This work presents experimental observations of strong expanding thermal jets following the application of nanosecond spark discharges. These jets propagate in a toroidal shape perpendicular to the interelectrode axis, with high velocities of up to $30 \mathrm{~m} / \mathrm{s}$ and over distances of centimeter scale. Their propagation length is much larger than the thermal expansion region produced by the conventional millisecond sparks used in car engine ignition, thus greatly improving the volumetric excitation of gas mixtures. The shape and velocity of the jets is found to be fairly insensitive to the shape of the electrodes. In addition, their spatial extent is found to increase with the number of nanosecond sparks and with the discharge voltage, and to decrease slightly with the pressure between 1 and 7 atm at constant applied voltage. Finally, this thermal jet phenomenon is observed in experiments conducted with many types of gas mixtures, including air, nitrogen, argon, and combustible $\mathrm{CH}_{4} /$ air mixtures. This makes Nanosecond Repetitively Pulsed (NRP) discharges particularly attractive for aerodynamic flow control or plasma-assisted combustion because of their ability to excite very large volumes of gas.
\end{abstract}

\section{Introduction}

Nanosecond discharges have shown strong potential for various applications such as PlasmaAssisted Ignition (PAI) [1-5], lean flame stabilization [6-8], aerodynamic flow control [9-12] and nanomaterial synthesis [13-15].

In air-containing mixtures, the phenomenon of ultrafast gas heating by nanosecond high voltage (HV) pulses was first experimentally observed in [16]. This phenomenon was shown to be caused by dissociative quenching reactions between molecular oxygen and electronically excited nitrogen molecules produced by electron impact during the discharge $[17,18]$. This effect was also predicted numerically [19-21]. The ultrafast gas heating in atmospheric pressure air plasmas occurs within a few tens of nanoseconds following a nanosecond spark pulse, and it also leads to the nearly full dissociation of molecular oxygen [18,22-24] thus producing large amounts of reactive chemical species and making NRP discharges attractive for the applications mentioned above.

An expected consequence of the ultrafast gas heating following nanosecond sparks is the formation of shock waves and hydrodynamic effects. Some of the earliest experimental 
observations and numerical modeling of these effects for a short duration spark of a few mJ were presented in [25]. The authors observed that the hot gas produced by a single spark propagated over a few millimeters within tens of microseconds. In further developments, Leonov et al [26] investigated high current ( $1.5 \mathrm{kA}$ ) sparks of 50 -ns duration across long interelectrode gaps (50$60 \mathrm{~mm}$ ) in air, and observed the onset of strong turbulence attributed to Rayleigh-Taylor instabilities, with a radial expansion of the hot gas core to distances up to 50 times the radius of the initial spark channel. More recently, this effect was also observed in nanosecond sparks with lower current ( 100 A)[27-30]. Xu et al [31,32] further showed that the hydrodynamic effects induced by a burst of nanosecond pulses in high pressure, lean propane-air mixtures can cause wrinkling of the flame front and accelerate flame propagation. This was also observed by Bane et al [33].

Insights into the mechanism responsible for the onset of these wrinkled combustion front were recently proposed by Castela et al [35] who demonstrated, through a comparison of experimental results and numerical modeling, that the hot gas core produced by the spark develops into a torus of advected reactive hot gas, and that fresh gas recirculates inside the interelectrode region. This was confirmed by Schlieren diagnostics in [27] and by PIV (particle image velocimetry) measurements in [36].

In this paper, we present a detailed investigation of the expanding hot gas core produced by single nanosecond discharges and by bursts of nanosecond discharges. The motion of the hot gas core is followed up to the millisecond time scale. We present a parametric study of the effects of various parameters, namely the number of nanosecond sparks, applied voltage amplitude, shape of the electrodes, and gas composition, on the hydrodynamic effects induced by nanosecond sparks.

\section{Experimental}

Nanosecond Repetitively Pulsed (NRP) discharges were produced between two metallic pin electrodes separated by a distance of $1 \mathrm{~mm}$. Most experiments reported here were performed with paraboloid pins with tapered $150-\mu \mathrm{m}$ tip radius, but additional experiments were conducted with conical or cylindrical pins. The NRP discharges were generated with an FID FPG 30100MC4K pulser producing pulses of 10-ns duration, amplitudes up to $42 \mathrm{kV}$ and Pulse Repetition Frequency (PRF) up to $100 \mathrm{kHz}$. The energy deposited into the plasma was measured with a high voltage probe (Lecroy PPE $20 \mathrm{kV}$ ), current probe (Pearson model 6585) and recorded on a LeCroy Wavepro 7100 oscilloscope. A description of technique used for energy measurements can be found elsewhere $[16,18]$.

To characterize the hydrodynamic effects induced by the spark up to $1 \mathrm{~ms}$ after the discharge, phase-locked Schlieren measurements were performed with the experimental setup, similar to the one used in [28], shown in Figure 1. The gate of the ICCD PIMAX camera used for the Schlieren measurements was set to $0.1 \mu \mathrm{s}$. Fast imaging of spontaneous emission of $\mathrm{OH}^{*}(308 \mathrm{~nm})$ was recorded with a Photron SA-X2 camera, using a camera gate of $5 \mu$ s. 
To ensure a complete characterization of the gas density gradients induced by the discharge, the knife-edge of the Schlieren setup was placed either horizontally or vertically. In the horizontal position (i.e. parallel to the interelectrode axis), the knife-edge cuts the light deflected toward the bottom side of the image; in the vertical position (i.e. perpendicular to the interelectrode axis), it cuts the light deflected toward the right side of the image. For most Schlieren images presented here, the knife-edge was set to cut about $90 \%$ of the light (perpendicular to the interelectrode axis) in order to increase contrast.

\section{Results}

\section{Gas recirculation and transport of active speciesfollowing a single nanosecond spark}

Figure 2 presents a set of Schlieren images obtained from 10 to $300 \mu$ s after a single nanosecond spark. The discharge was initiated in ambient air by a high voltage (HV) pulse of 36-kV amplitude and 10-ns duration. Figure 2 shows that the hydrodynamic disturbance starts within a few microseconds after the discharge. Two main phenomena can be observed: shock wave propagation during the first $\sim 10 \mu \mathrm{s}$, and expansion of the hot gas core up to $300 \mu \mathrm{s}$. The shock waves induced by nanosecond discharges have been observed before $[27,37,38]$. The subsequent expansion of the hot gas shows how the energy initially deposited into the interlectrode discharge channel redistributes spatially. The Schlieren results presented in Figure 2 show that the shape of the heated gas region is symmetrical around the pin axis, and that the hot gas expands by about 6 $\mathrm{mm}$ within $300 \mu \mathrm{s}$. So, the energy initially deposited into a thin nanosecond spark channel (1$\mathrm{mm}$ length and $100-\mu \mathrm{m}$ diameter) redistributes over a large volume of $6-\mathrm{mm}$ diameter within about $100 \mu$ s.

Figure 3 presents Schlieren images for the same conditions as in Figure 2 but with the knife-edge placed vertically in order to visualize the gas density gradients in the direction parallel to the pin axis. The combination of the data presented in Figures 2 and 3 provides a full map of the hot gas region and evidences the gas recirculation pattern between the pin electrodes: after about $30 \mu \mathrm{s}$ the hot gas and active species created by the discharge between the electrodes are transported outwards in a toroidal jet and the interelectrode gap is refilled by fresh gas. This recirculation effect was already reported by Kono [25] and Maly [39]. Two-dimensional modeling by Thiele, Warnatz et al [40] describes the mechanism of gas recirculation. Recently, three-dimensional Direct Numerical Simulations (DNS) of the hydrodynamic effects induced by a nanosecond spark for the conditions considered in the present work were performed by Castela et al [35]. These DNS simulations confirm the creation of a torus of ejected hot gas and the recirculation of fresh gases along the interelectrode axis.

Figure 4 compares a Schlieren image and the distribution of $\mathrm{OH}^{*}(308 \mathrm{~nm})$ spontaneous emission intensity at the same instant $(30 \mu \mathrm{s})$. The discharge conditions for Figure 4 are the same as for Figures 2 and 3. The distributions of gas density perturbation and of $\mathrm{OH}^{*}$ emission 
intensity are found to have the same toroidal shape and radius. This indicates that reactive species are transported in the hot torus.

The blue curve in Figure 4c presents the evolution of the diameter $d$, defined in Figure 4a, of the hot gas torus produced by a single nanosecond spark. One can see that the hot gas torus first expands rapidly ( $d d / \mathrm{d} t \sim 60 \mathrm{~m} / \mathrm{s}$ ) and then slows down after $\sim 100 \mu \mathrm{s}$. The energy deposited in the plasma is thus redistributed into a volume of characteristic scale $d_{\max }$ in approximately $100 \mu \mathrm{s}$.

The red curve in Figure 4c presents the expansion of the hot gas after ignition by a spark produced by a conventional automotive ignition coil with energy of about $54 \mathrm{~mJ}$ and charging duration of $4 \mathrm{~ms}$. The hot gas torus induced by the nanosecond spark expands significantly faster and further than that produced by the conventional millisecond spark, even though the energy deposited by the conventional spark $(\sim 54 \mathrm{~mJ})$ is much higher than that of the nanosecond spark ( $6 \mathrm{~mJ})$. The faster gas motion in the case of nanosecond spark is explained by the higher temperature gradients following the short (10 ns) energy deposition.

\section{Parametric studies}

The following sections examine the dependence of the speed and spatial extent of the hot gas torus expansion as a function of shape and material of the electrodes, gas composition, applied pulse voltage amplitude, and number of pulses in the case of multiple nanosecond sparks.

\section{i) Shape of the electrodes and their material}

Figure 5 presents Schlieren images obtained after a single nanosecond spark in air $(U=36 \mathrm{kV}$, $P=1$ atm, 10 ns pulse) with different electrode systems: a) conic stainless steel pins with $50 \mu \mathrm{m}$ tip; b) parabolic tungsten pins tapered with $150 \mu \mathrm{m}$ tip radius; c) cylindrical tungsten electrodes of $2 \mathrm{~mm}$ diameter. All images were taken $300 \mu$ s after the discharge. It can be seen that the scale and the shape of the expanding hot gas core are nearly identical in all three cases, thus the observed phenomenon does not depend on the electrode shape or the material used here.

The effects of the electrode shape were investigated by Bane et al[33] who presented Schlieren images taken after a nanosecond spark in an $\mathrm{H}_{2}$ /air mixture at $P=1$ atm. These authors demonstrated that factors such as the heat exchange between the electrodes and the hot gas or viscous effects may play a significant role in the energy balance of the system and affect the initiation of the ignition kernel. However, as shown above, the spatial scale of the energy redistribution is practically the same for very different electrode geometries investigated here. It should be also noted that the results of calculations for nanosecond spark presented in [35] demonstrate that the effect of gas recirculation that governs the spatial energy redistribution is not induced or affected significantly by presence of electrodes (or their shape) near the plasma. 


\section{ii) Single nanosecond pulse vs burst of nanosecond pulses}

For applications of nanosecond discharges in repetitive mode (NRP) in plasma assisted combustion and flow control, it is important to understand how the hot gas core expansion and fresh gas recirculation are affected by multiple sparks. The hot core expansion process depends on the value of the energy density deposited by the discharge, and this parameter strongly depends on the initial gas number density in the space between the electrodes. When a burst of pulses is applied, it is often considered that the gas density before each new pulse is lower than before the previous one because the gas heated by previous pulses diffuses slowly. If this were the case, then the highest gas temperature would always be between the electrodes and each new discharge would be initiated in the hot gas with lower number density. However, we have shown that the energy deposited into plasma does not remain confined between the electrodes, but spatially redistributes owing to the motion of the hot gas torus. If the period between nanosecond pulses is longer than the recirculation time of the fresh gas, then each new discharge will be triggered at the same conditions as the first one, i.e. in the fresh gas. If the period between the nanosecond pulses is shorter than the flow recirculation time, then all discharges after the first one will be initiated at higher temperature, i.e. at lower gas number density. This means that the energy deposited in each new pulse will significantly change at some critical frequency.

Figure 6a,b presents the comparison of a single pulse discharge in air at $P=1 \mathrm{~atm}, U=28 \mathrm{kV}$ with the sequence of discharges initiated by a burst of 30 pulses at $f=70 \mathrm{kHz}, U=28 \mathrm{kV}$. Both images were obtained $500 \mu$ s after the first pulse. The shape of the expanded hot gas in the case of multiple nanosecond pulses is seen to be a superposition of the toruses produced by individual pulses. However, we note a strong synergy between pulses, as the torus produced by a given pulse appears to be pushed outwards by the previous pulses. One can see that the size of the hot gas core is much larger for the burst of sparks. Furthermore, Figure 6c shows that the spatial scale of the hot gas torus depends linearly on the number of the pulses in a burst, i.e. linear to the energy deposited in plasma. This means that the volume of the hot gas core grows disproportionally large with energy deposited in plasma. The red dashed line in Figure 6c corresponds to the dimension of the hot gas at $500 \mu$ s after ignition of a standard automotive spark. As can be seen from Figure 6c, the spatial extent of the gas region heated by a single pulse of energy $2.5 \mathrm{~mJ}$ is already much larger than that produced by the standard automotive spark, and it extends even further when multiple nanosecond pulses are applied.

\section{iii) Dependence on gas composition, pressure and applied voltage}

We now present results on the expansion dynamics of the hot gas core in various gases, namely air, nitrogen, argon, and a stoichiometric $\mathrm{CH}_{4} /$ air mixture. A single 10-ns discharge was initiated for all cases at $P=1 \mathrm{~atm}, U=36 \mathrm{kV}$ at 1-mm gap between the pin-to-pin electrodes. As can be clearly seen in Figure 7a, the dynamics of the expansion as well as the maximal propagation length are similar for all gases considered here. We note that the maximal radius is slightly larger 
for the two mixtures containing air molecules, which may be attributed to the stronger effect of ultrafast heating via dissociative quenching of excited $\mathrm{N}_{2}$ with $\mathrm{O}_{2}$.

Figure 7b presents the dependence of $d_{\max }$ on pressure in the range 1-7 atm for a single nanosecond spark and for a burst of 10 sparks (10 ns each) applied at a frequency of $70 \mathrm{kHz}$. The applied voltage is $U=36 \mathrm{kV}$ for all pulses, and the interelectrode gap is fixed at $1 \mathrm{~mm}$. The increase of pressure slightly decreases the maximal propagation length $d_{\max }$ for both the single spark and the burst of sparks. Interestingly, at 7 atm there is practically no difference in maximum propagation length between the single spark and the burst. This suggests that the multiple toruses merge into a single torus of high cumulative energy.

Finally, Figure 7c presents the dependence of $d_{\max }$ vs applied voltage for a single spark and for multiple sparks. For both single and multiple sparks, the maximal propagation length of the hot gas core increases up to 50 percent over the voltage range considered here.

Based on the foregoing observations, it is clear that $d_{\max }$ can be significantly increased at sub millisecond scale by increasing the number of multiple nanosecond sparks and by increasing their voltage amplitude. This provides a convenient and effective way to control the spatial distribution of the energy deposited in the plasma, which is promising for ignition of lean combustible mixtures in volume.

\section{Discussion and conclusions}

We have shown that the energy deposited by single nanosecond spark is initially concentrated in a thin hot channel about $100 \mu \mathrm{m}$ in diameter (see Figure 2). Then this hot gas expands and forms a torus whose diameter increases up to about $5 \mathrm{~mm}$ within about $100 \mu \mathrm{s}$ and then slows down. This expansion of the hot gas is more than twice higher than for the conventional spark even though the energy deposited in a single ns spark is almost 10 times lower than in the conventional spark. The advected hot gas also contains the active species produced by the spark discharge. Fresh gas is drawn into the interlectrode gap, thus creating a strong recirculation zone. This mechanism has an interesting effect of redistributing the energy deposited by the discharge into a very large volume of gas within a very short time.

This phenomenon is shown to occur in different gases (Air, $\mathrm{CH}_{4} /$ Air, $\mathrm{N}_{2}$, Ar) over a wide range of pressures (1-7) atm, voltage amplitudes (6-40) $\mathrm{kV}$ and to be almost independent on the shape and material of the pin electrodes. The temporal dynamics of the gas expansion after a single nanosecond spark were found to be practically the same for all considered conditions and for all gases investigated.

Furthermore, the experiments conducted with multiple nanosecond sparks demonstrated that the dimension of the expanded hot gas torus increases linearly with the number of multiple sparks. It is therefore possible to affect a large gas volume (torus of radius $>10 \mathrm{~mm}$ ), thus making NRP 
discharges a promising tool for stabilization or ignition of lean combustible mixtures and aerodynamic flow control.

\section{Acknowledgments}

This work was supported by the FAMAC (Grant no. ANR-12-VPTT-0002) and PLASMAFLAME (Grant no.ANR-11-BS09-0025) projects. The authors thank Dr. Denis Packan(ONERA) for providing the Schlieren light source.

\section{References}

[1] Pancheshnyi S et al 2006 IEEE Transactions on Plasma Science 34 2478-2487

[2] Singleton D et al 2011 J. Phys. D: Appl. Phys. 44022001

[3] Starikovskiy A et al 2013 Prog. Energy Combust. Sci. 39 61-110

[4] Starikovskaia S 2014 J. Phys. D: Appl.Phys. 47353001

[5] Ju Y et al 2015 Progress in Energy and Combustion Science 48 21-83

[6] Barbosa S et al 2015 Phil. Trans. R. Soc. A 37320140335

[7] Pilla G et al 2006 IEEE Transactions on Plasma Science 34 2471- 2477

[8] Hyungrok D et al 2008 IEEE Transactions on Plasma Science 36(6) 2898-2904

[9] Miles R et al 2009 Eur. Phys. J. Appl. Phys. 4722802

[10] Benard N et al 2008 AIAA Journal 46 (9) 2293-2305

[11] Adamovich I et al 2009 Plasma Sources Sci. Technol. 18034018

[12] Opaits D 2010 Ph.D. Dissertation, Princeton University

[13] Pai D et al 2013 Scientific Reports 31221

[14] Chen Z et al. 2011 Nano Lett. 11 4168-4175

[15] Ostrikov K 2011 J. Phys. D-Appl. Phys. 44174003

[16] Pai D et al 2010 Plasma Sources Science and Technology 19065015

[17] Stancu G et al 2010 J. Phys. D: Appl. Phys. 43124002

[18] Rusterholtz D et al 2013 J. Phys.D: Appl. Phys 46464010

[19] Popov N 2001 Plasma Physics Reports 27(10) 886-896

[20] Flitti A et al 2009 Eur. Phys. J. Appl. Phys. 4521001

[21] Aleksandrov 2010 J. Phys. D: Appl. Phys 43255201

[22] Stancu G et al 2010 J. Phys. D: Appl. Phys.43 124002

[23] Lo A et al 2012 Appl Phys B Lasers O 107(1) 229-242

[24] Klochko et al 2015 Plasma Sources Science and Technology 24(2) 025010 
[25] Kono M et al 1989 Symp. (Int.) Combust. 221643

[26] Leonov S et al 2007 Physics of Plasmas 14123504

[27] Xu D et al 2011 Appl. Phys. Lett. 99121502

[28] Xu D et al 2014 J. Phys. D: Appl. Phys. 47235202

[29] Lo A et al 2014 Journal of Physics D: Applied Physics 47115201

[30] Lo A et al 2014 Journal of Physics D: Applied Physics 47115202

[31] Xu D 2013 PhD thesis December, Ecole Centrale, Paris

[32] Xu D et al 2016 Plasma Chemistry and Plasma Processing 36 (1) 309-327

[33] Bane S et al 2015 Combustion and Flame 162(2) 462-469

[34] Xu D et al 2016 Plasma Chem Plasma Process 36 309-327

[35] Castela M et al 2016 Proceedings of the Combustion Institute 000 1-9

[36] Seydou A et al 2016 18th International Symposium on the Application of Laser and Imaging Techniques to Fluid Mechanics, Lisbon, Portugal, july 4-7

[37] Nagaraja S et al 2014 Combustion and Flame 161 1026-1037

[38] Starikovskii A et al 2009 Plasma Sources Science and Technology 18(3)

[39] Maly R 1984 Fuel Economy in Road Vehicles Powered by Spark Ignition Engines, Springer US, p. 91

[40] Thiele M et al 2000 Proceedings of the Combustion Institute 28 1177-1185

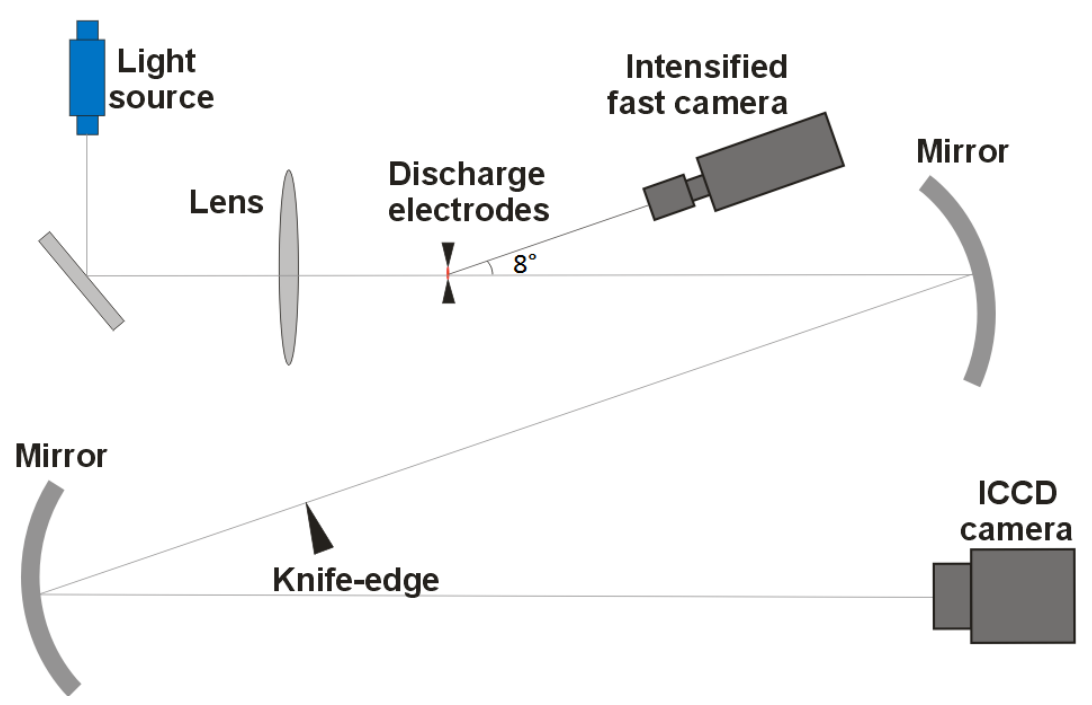

Figure 1. Experimental setup. The focal length of the lens is $1 \mathrm{~m}$. Both spherical mirrors have focal distance of $2 \mathrm{~m}$. 


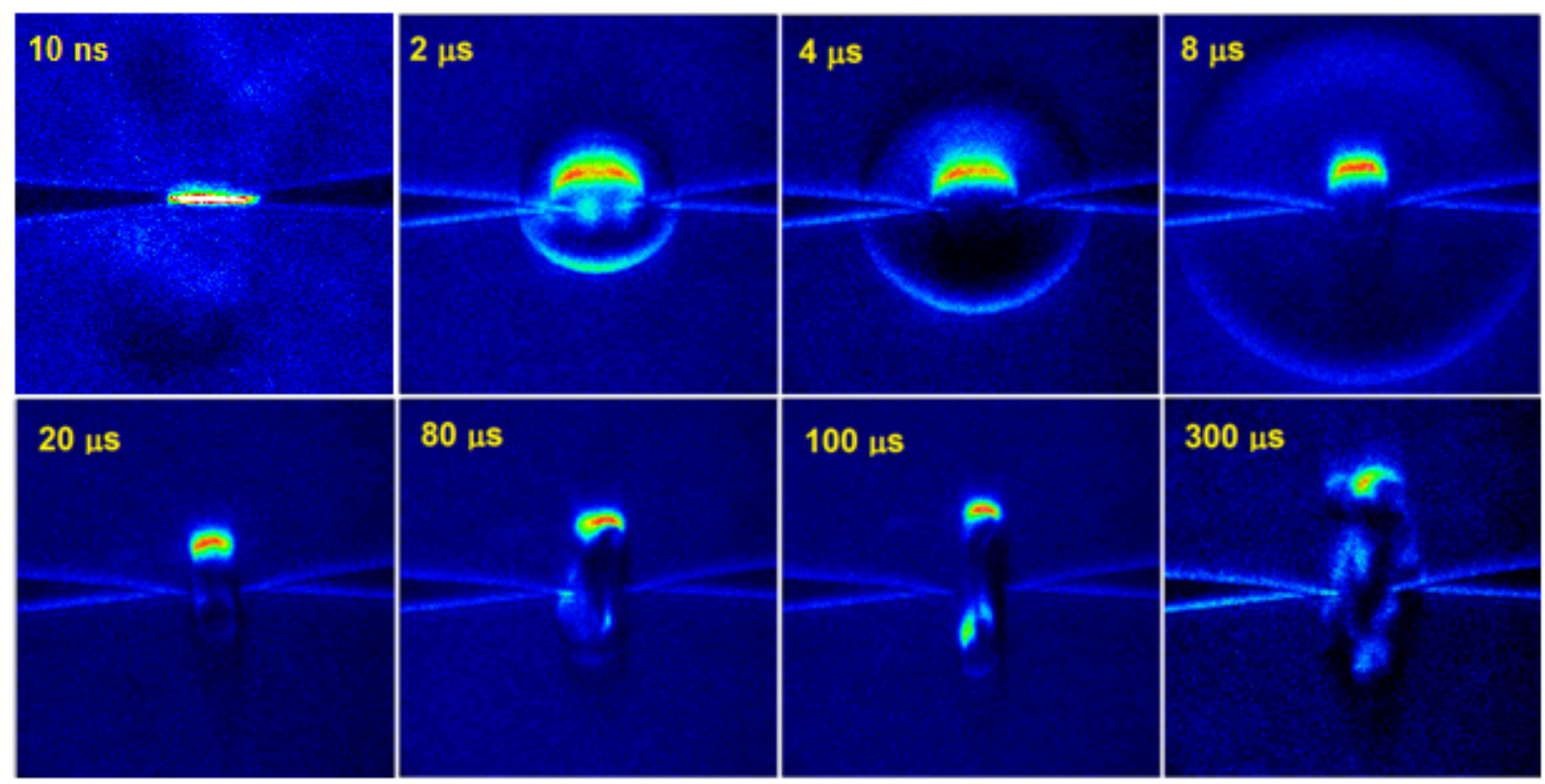

Figure 2. Schlieren images obtained for a single nanosecond spark in air at $P=1 \mathrm{~atm}, U=36 \mathrm{kV}$. The distance between the pins is $1 \mathrm{~mm}$. The knife edge is horizontal and cuts $90 \%$ of the light

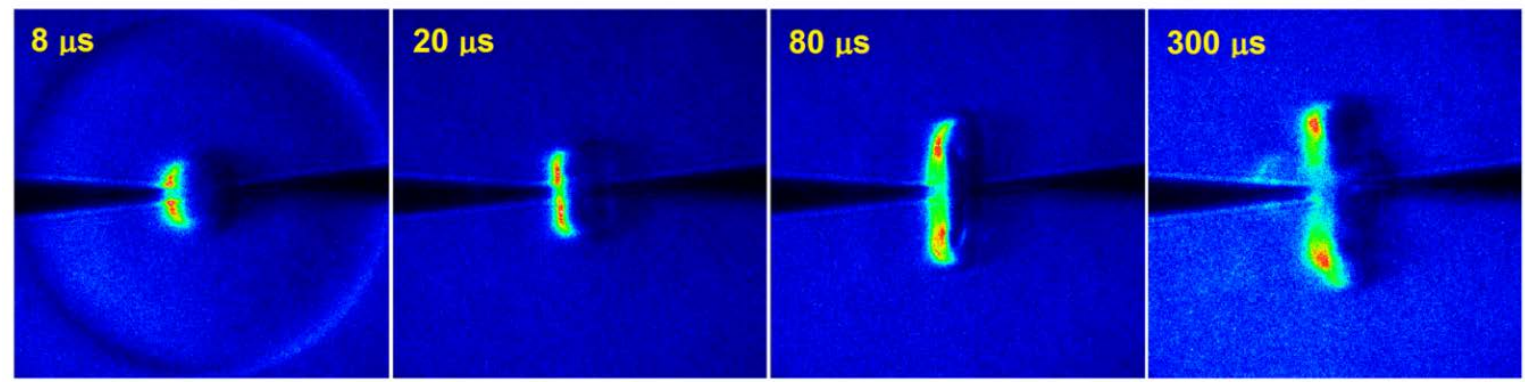

Figure 3. Schlieren images obtained for a single nanosecond spark in air at $P=1 \mathrm{~atm}, U=36 \mathrm{kV}$. The distance between the pins is $1 \mathrm{~mm}$. The vertical knife edge cuts $90 \%$ of the light.

(a) Schlieren image

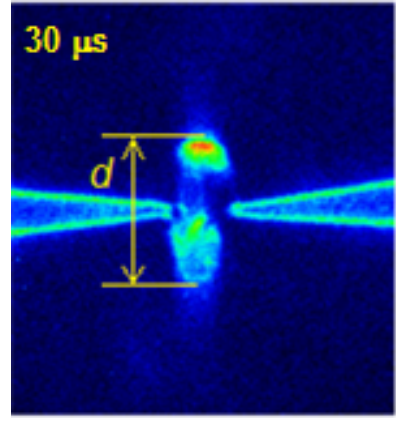

(b) $\mathrm{OH}^{*}$ emission

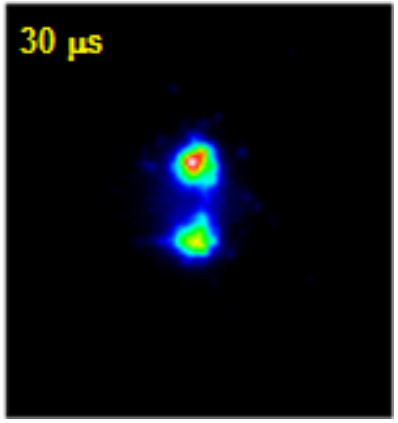

(c) Evolution of the hot diameter

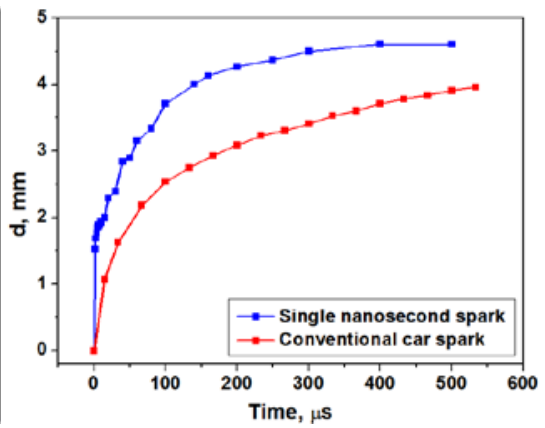

Figure 4. Comparison of a) Schlieren image and b) distribution of spontaneous $\mathrm{OH}^{*}$ emission (308 nm) at $30 \mu \mathrm{s}$ after a single nanosecond spark in air at $P=1 \mathrm{~atm}$, with a 10 ns pulse of amplitude $U=36 \mathrm{kV}$, $\varepsilon=6 \mathrm{~mJ}$; c) Evolution of the hot core diameter $d$; Nanosecond spark (blue curve): $U=36 \mathrm{kV}, 10$ ns pulse, deposited energy: $\varepsilon=6$ mJ. Conventional automotive spark (red curve): $\varepsilon=55 \mathrm{~mJ}$. Distance between pins: $1 \mathrm{~mm}$. 
(a)

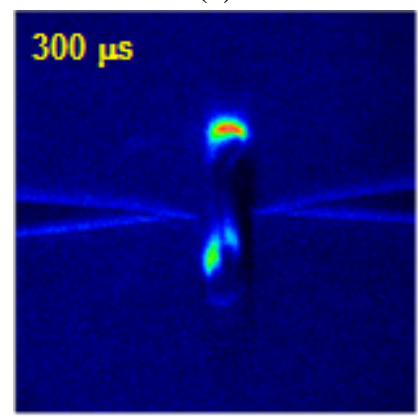

(b)

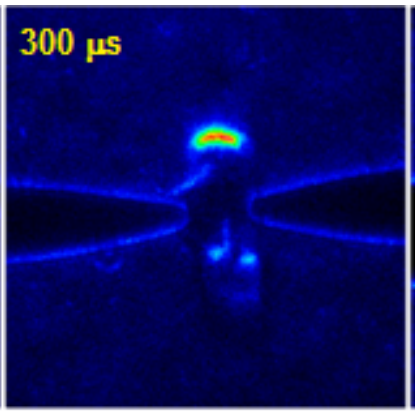

(c)

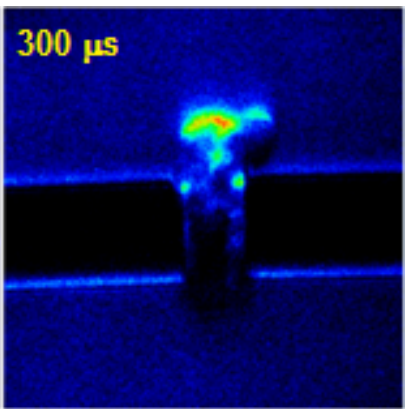

Figure 5. Schlieren images obtained for a single nanosecond spark in air at $P=1 \mathrm{~atm}, U=36 \mathrm{kV}$ for different electrode systems: a) conic stainless steel pin with $30-\mu$ m radius of curvature tips; b) paraboloid tungsten pins with tapered $150-\mu \mathrm{m}$ tip radius; c) cylindrical tungsten electrodes of diameter $2 \mathrm{~mm}$. The distance between the electrodes is $1 \mathrm{~mm}$. The horizontal knife edge cuts $90 \%$ of the light.

(a) Single pulse

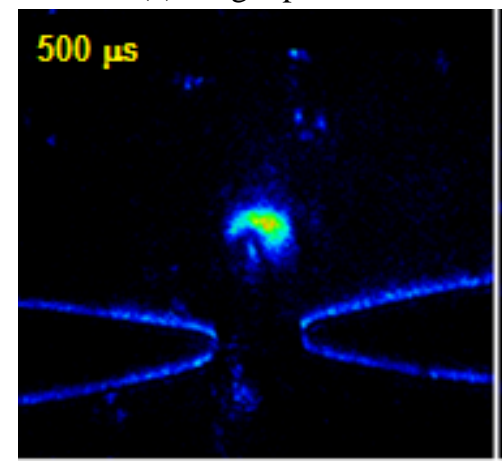

(b) Burst of 30 pulses

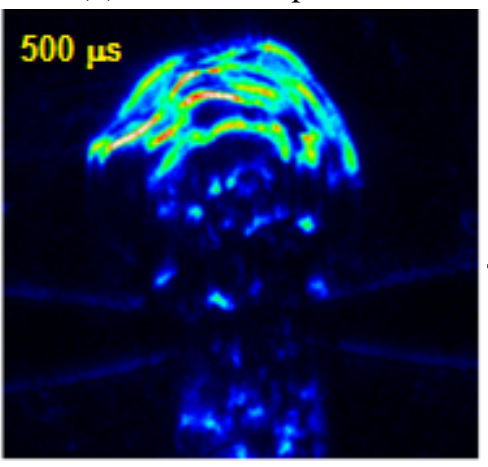

(c) Evolution of hot diameter

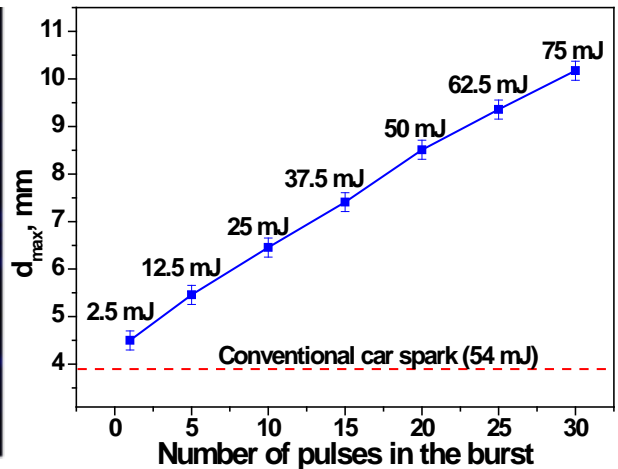

Figure 6. Schlieren results obtained at $500 \mu$ s after the discharge in air at $P=1 \mathrm{~atm}, U=28 \mathrm{kV}$ and $1 \mathrm{~mm}$ distance between the electrodes; a) single pulse; b) burst of 30 pulses at $f=70 \mathrm{kHz}$; c) Dependence of the hot gas core size $d_{\max }$ at $500 \mu \mathrm{s}$ on the number of pulses at $70 \mathrm{kHz}$. The red dashed line represents the expansion size for the conventional automotive spark.

(a) Different gas mixtures

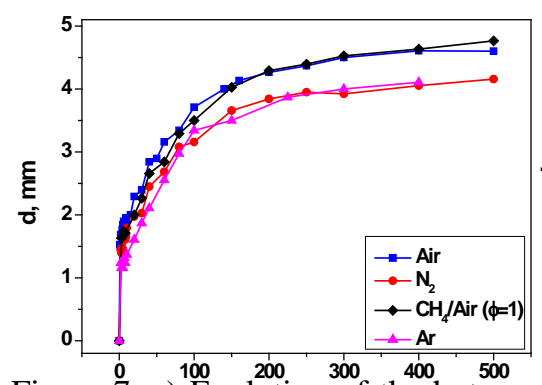

(b) Different gas pressures

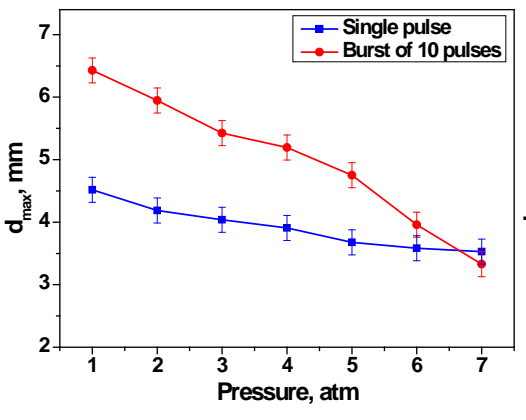

(c) Different applied voltage

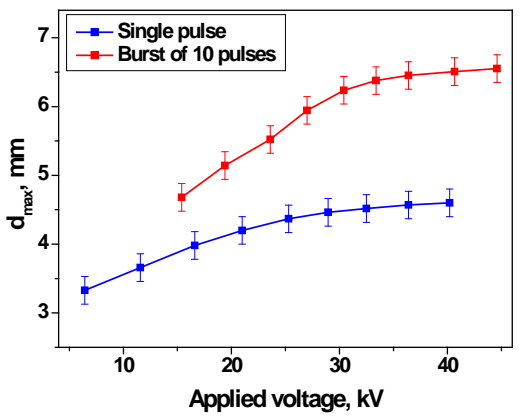

Figure 7. a) Evolutitime, ${ }^{300} \mathrm{f}$ the hot core size diameter $d$ after a single nanosecond spark in Air, $\mathrm{N}_{2}, \mathrm{Ar}$ and $\mathrm{CH}_{4} /$ Air stoichiometric mixture. $P=1 \mathrm{~atm}, U=36 \mathrm{kV}$; b) Dependence of the hot core size $d_{\max }($ at $t=500 \mu \mathrm{s}) v s$ pressure $P$ for a single ns spark and a burst of 10 pulses at $f=70 \mathrm{kHz}$. Air, $U=28 \mathrm{kV}$; c) Evolution of the hot core size $d_{\max }$ (at $t=500$ $\mu$ s) after a single ns spark and a burst of 10 pulses ( $f=70 \mathrm{kHz}, U=28 \mathrm{kV}$ ) at different amplitudes of applied voltage; For all figures the duration of the single pulse is $10 \mathrm{~ns}$ and the distance between the electrodes is $1 \mathrm{~mm}$. 